\title{
Interaction Energy Analysis for Drug-Cyclodextrin Inclusion Complexes in Aqueous Solutions
}

\author{
Masao Fujisawa ${ }^{1, *}$, Takanobu Yasukuni ${ }^{1}$, Hirohito Ikeda ${ }^{2}$, Miho Yukawa ${ }^{2}$, Hatsumi Aki $^{2}$ and \\ Takayoshi Kimura $^{3}$
}

${ }^{1}$ Kinki University, Department of Biotechnological Science, Kinokawa-City, Wakayama 649-6493, Japan

${ }^{2}$ Fukuoka University, Department of Pharmaceutical Science 814-0180, Fukuoka, Japan

${ }^{3}$ Kinki University, Department of Chemistry, Higashiosaka Osaka 577-8501, Japan

\begin{abstract}
It is vital to elucidate the role of asymmetric intermolecular interactions resulting from the stereospecific structures of molecules in order to understand the mechanisms of chemical and biochemical reactions such as enzymesubstrate reactions, antigen-antibody reactions, etc. In order to reveal the mechanism of the inclusion phenomenon for $\beta$-cyclodextrin (CD)-ampicillin complexes and $\beta$-CD-ibuprofen complexes, binding free energies were determined using molecular mechanics/Poisson-Boltzmann surface area (MM/PBSA) analysis. To clarify the details of the interaction energies of these complexes, pair interaction energy decomposition analysis (PIEDA) was carried out. The direction of inclusion of drugs into $\beta-C D$ cavities was clarified on the basis of results obtained using the above-mentioned methods.
\end{abstract}

Keywords: Binding free energy, energy decomposition, drug-cyclodextrin complex, dispersion force, interaction energy.

\section{INTRODUCTION}

Molecular recognition and discrimination may be caused by the interactions among containing surfaces of colliding molecules in solutions and mixtures. In particular, stereospecific interactions due to neighboring surfaces may play the leading role in, for example, enzyme-substrate reactions, antigen-antibody reactions, some types of mechanisms of the senses of smell and taste, etc. Therefore, it is vital to elucidate the role of asymmetric intermolecular interactions due to the stereospecific structure of a molecule in order to understand the mechanisms of chemical and biochemical reactions. In previous research, thermodynamic functions for the molecular inclusion of simple molecules into $\alpha$ - and $\beta$-cyclodextrin (CD) cavities in dilute aqueous solutions were systematically determined using microcalorimetry in order to clarify the mechanism of molecular recognition and discrimination [1-10]. Improvements in the stability and solubility of drugs can be expected from formation of CD-drug inclusion complexes. Moreover, complexation of $C D$ with drugs can prevent aggregation of drugs. Therefore, consideration of the interaction energy using energy decomposition analysis is required as basic information for understanding the mechanisms of CDdrug complexation. For the $C D$ system of a pharmaceutical drug, theoretical calculations of

*Address corresponding to this author at the Kinki University, Department of Biotechnological Science, Kinokawa-City, Wakayama 649-6493, Japan; Tel: +81-736-770345 Ext. 2211; Fax: +81-736-774754;

E-mail: fujisawa@waka.kindai.ac.jp molecular interactions of the complex $(\beta-C D+$ chlorambucil) were carried out using the molecular orbital method. The correlation between energy changes and molecular structures were examined. The large interaction energies calculated by the molecular orbital method showed the inclusion phenomenon [11]. However, the major contributions to the interaction energy were not discussed in detail. In addition, the direction from which ampicillin enters the $C D$ cavity could not be clearly concluded using the semiempirical molecular orbital method and the NMR spectrum [12].

In this study, we were interested in clearly detailing the major contribution to the interaction energy of CDs and guest molecules. The interaction energies for the inclusion complexation of the $\beta$-lactam antibiotic ampicillin and the nonsteroidal anti-inflammatory drug ibuprofen with $\beta-C D$ in aqueous solutions were analyzed by molecular mechanics/Poisson-Boltzmann surface area (MM/PBSA) analysis [13] and pair interaction energy decomposition analysis (PIEDA) [14]. Predictions regarding the direction of inclusion of drugs into the cavity of $\beta-C D$ were made on the basis of the computational results.

\section{COMPUTATIONAL METHODS}

\subsection{MM/PBSA Analysis}

A conformation search was performed with the MMFF94s force field using CONFLEX 6 [15] to determine the direction from which drugs enter the $\beta$ $C D$ cavity. Ampicillin has a lactam ring and a phenyl 
ring, whereas ibuprofen has an isobutyl group and propanoic acid as shown in Figure 1. It was clear that these functional groups cannot insert from the primary hydroxyl side of $\beta-C D$ and it was confirmed that they can insert from the secondary side of $\beta-C D$. We examined two conformations for each CD-drug complex as follows: (3a) the lactam ring of ampicillin exists inside the $\beta-C D$ cavity while the phenyl ring exists outside on the secondary hydroxyl side of $\beta-C D$ (Lact_BCDAmp); (3b) the phenyl ring of ampicillin exists inside the $\beta-C D$ cavity while the lactam ring exists outside on the secondary hydroxyl side $\beta-C D$ (Phen_BCDAmp); (4a) the isobutyl group of ibuprofen exists inside the $\beta-C D$ cavity while the propanoic acid exists outside on the secondary hydroxyl side of $\beta-C D$ (Isob_BCDIbup); and (4b) the propanoic acid of ibuprofen exists inside the $\beta-C D$ cavity while the isobutyl group exists outside on the secondary hydroxyl side of $\beta-C D$ (Prop_BCDlbup). The optimization and electrostatic potential calculations of these conformations were conducted using Gaussian 09 Revision C.01 [16]. The partial charges of all molecules were fitted with $H F / 6-31 G(d)$ calculations and the RESP module in the AMBER package [17], and were generated automatically using the antechamber program in AMBER 12.0.

In molecular mechanics (MM) minimizations and molecular dynamics (MD) simulations, the general AMBER force field (GAFF) [18] was used for CD and the guest molecules. The solvated systems were equilibrated by carrying out 1000 short steps of minimizations, and 10 ps of heating and constant pressure equilibration at $300 \mathrm{~K}$. All simulations were run with shake on hydrogen atoms, a 2 fs time step, and Langevin dynamics for temperature control. We ran a total of $20 \mathrm{~ns}$ or production recorded the coordinates every $10 \mathrm{ps}$. This was run 4 times to obtain 20 ns of simulation time. An average structure of each



Ampicillin conformation was created over our entire trajectory of the last 5 ns.

In MM/PBSA, the binding free energy $\left(\Delta G_{\text {bind }}\right)$ between $C D$ and a drug to form a complex was calculated as:

$$
\begin{aligned}
& \Delta G_{\text {bind }}=G(\text { complex })-G(\beta-C D)-G(\text { drug }) \\
& \Delta G_{\text {bind }}=\Delta E_{\mathrm{MM}}+\Delta G_{\text {solv }}-T \Delta S \\
& \Delta E_{\mathrm{MM}}=\Delta E_{\text {internal }}+\Delta E_{\mathrm{vdw}}+\Delta E_{\text {els }}
\end{aligned}
$$

where $\Delta E_{\mathrm{MM}}, \Delta G_{\mathrm{solv}}$, and $-T \Delta S$ denote the changes in the gas phase MM energy, solvation free energy, and entropy term upon binding, respectively. The term $\Delta E_{\mathrm{MM}}$ includes $\Delta E_{\text {internal }}$ (bond, angle, and dihedral), $\Delta E_{\text {els }}$ (electrostatic), and $\Delta E_{\mathrm{vdw}}$ (van der Waals) energies.

The entropy change upon guest binding, $-T \Delta S$, was computed by normal-mode analysis on a set of conformational snapshots taken from MD simulations. The term $\Delta G_{\text {solv }}$ was calculated as:

$\Delta G_{\text {solv }}=\Delta G_{\mathrm{PB}}+\Delta G_{\mathrm{SA}}$

where $\Delta G_{\mathrm{PB}}$ was calculated with the PoissonBoltzmann (PB) method and $\Delta G_{S A}$ was calculated from the solvent-accessible surface area.

The interaction energy, $\Delta G_{\text {gas }}$, between the complex, $\beta-C D$, and the drug in the gas phase was calculated as [19]:

$$
\begin{aligned}
& \Delta G_{\text {gas }}=G_{\text {gas }}(\text { complex })-G_{\text {gas }}(\beta-C D)-G_{\text {gas }}(\text { drug }) \\
& \Delta G_{\text {solv }}=\Delta G_{\text {solv }}(\text { complex })-\Delta G_{\text {solv }}(\beta-C D)-\Delta G_{\text {solv }}(\text { drug }) \\
& \Delta G_{\text {bind }}=\Delta G_{\text {gas }}+\Delta G_{\text {solv }}
\end{aligned}
$$

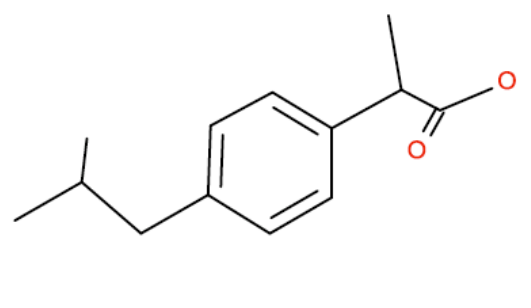

Ibuprofen

Figure 1: Structures of ampicillin and ibuprofen. 
where $\Delta G_{\text {solv }}($ drug $), \Delta G_{\text {solv }}(\beta-C D)$, and $\Delta G_{\text {solv }}$ (complex) are the solvation free energies of the drug, $\beta-C D$, and complex, respectively. The solvated monomer molecules mentioned in equation (6) are shown in Figure 2. In aqueous solution, the water molecules remaining in the $\beta-C D$ cavity are released into the bulk water upon inclusion of the drug.

\subsection{PIEDA}

PIEDA of the fragment molecular orbital method (FMO) was performed using the Gamess package [20] in order to evaluate the dispersion forces. An average structure of each conformation obtained using MM/PBSA was optimized at the RHF/6-31G level using the polarizable continuum model (PCM) with the integral equation formalism variant (IEFPCM). These structures were used for FMO calculations. PIEDA were decomposed into the electrostatic (ES), exchange-repulsion (EX), charge transfer (CT), and dispersion (DI) components, as shown in the following equation:

$\Delta E_{\text {int }}=\Delta E_{\mathrm{ES}}+\Delta E_{\mathrm{EX}}+\Delta E_{\mathrm{CT}}+\Delta E_{\mathrm{DI}}$

\section{RESULTS AND DISCUSSION}

\section{1. $\beta-C D+$ Ampicillin}

The binding free energies using MM/PBSA analysis are listed in Table 1. Lact_BCDAmp is more stabilized $\left(-47.1 \mathrm{~kJ} \mathrm{~mol}^{-1}\right)$ than Phen_BCDAmp. The structure in which the phenyl ring of ampicillin exists outside the $\beta$ CD cavity is stabilized compared with that in which the lactam ring exists outside the cavity. The average structure of Lact_BCDAmp determined using PMEMD indicates that Lact_BCDAmp forms an intermolecular hydrogen bond on the primary hydroxyl side of $\beta-C D$, and the $\mathrm{O}-\mathrm{H}$ distance is $0.09739 \mathrm{~nm}$, the $\mathrm{H} \cdots \mathrm{O}$ distance is $0.18749 \mathrm{~nm}$, and the $\mathrm{O}-\mathrm{H} \cdots \mathrm{O}$ angle is $168.739^{\circ}$, as shown in Figure $\mathbf{3 a}$. The lactam ring, which has large molecular volume, makes the closest contact with the atoms in the $\beta-C D$ cavity. On the other hand, as shown in Figure 3b. Phen_BCDAmp forms an intermolecular hydrogen bond on the secondary hydroxyl side of $\beta-C D$, and the $\mathrm{N}-\mathrm{H}$ distance is 0.10186 $\mathrm{nm}$, the $\mathrm{H} \cdots \mathrm{O}$ distance is $0.20447 \mathrm{~nm}$, and the $\mathrm{N}-\mathrm{H} \cdots \mathrm{O}$ angle is $173.985^{\circ}$. The molecular volume of the phenyl ring is not large and it cannot make close contact with the atoms in the $\beta-C D$ cavity. It seems that the difference in binding free energies was caused by forces between the ampicillin molecule and the inner walls of the $\beta-C D$ cavity for different conformations. The van deer Waals interactions of Lact_BCDAmp are greater than those of Phen_BCDAmp and the entropy decrease of Lact_BCDAmp is larger than that of Phen_BCDAmp. Lact_BCDAmp is stabilized significantly upon inclusion of ampicillin into the $\beta-C D$ cavity, which is accompanied by a large entropy decrease, and the stabilization is due to van deer Waals interactions resulting from the favorable fit. As
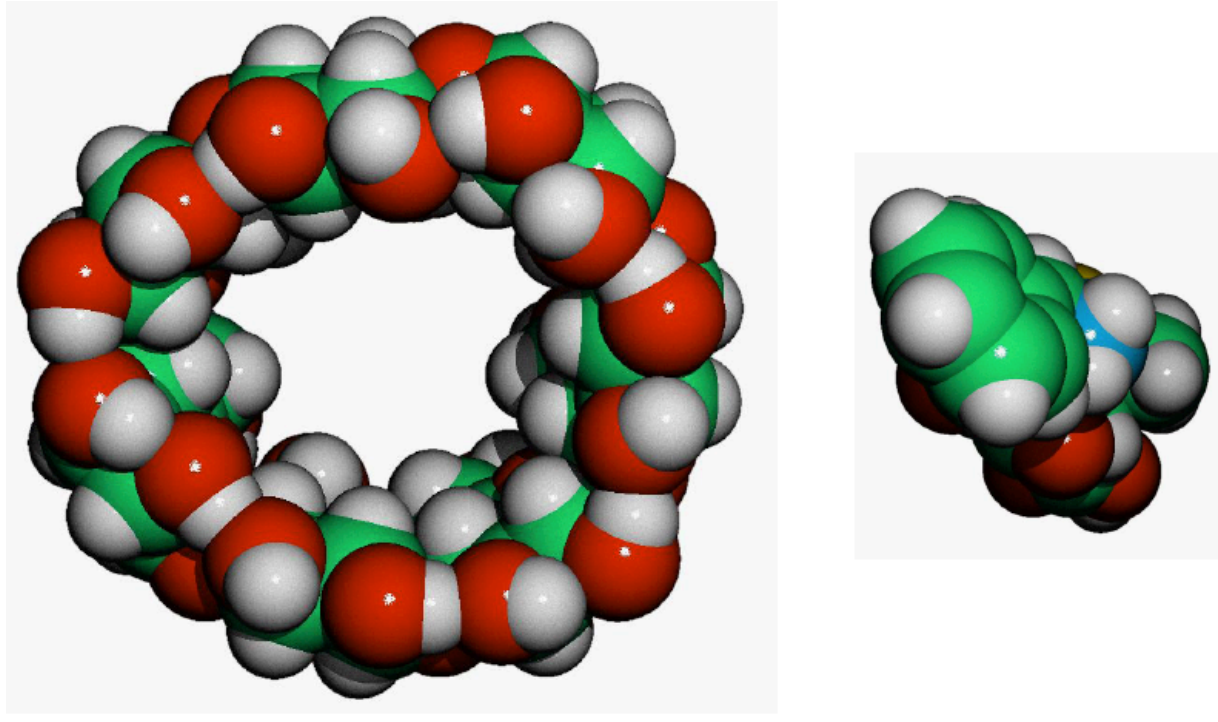

Figure 2: The solvated structures ampicillin and $\beta-C D$ used in solvation free energy calculations. 
Table 1: Binding Free Energies Predicted on the Basis of MM/PBSA Analysis for the Systems of $\beta-C D+$ Drug. All Data are Given in kJ mol-1

\begin{tabular}{|c|c|c|c|c|c|c|}
\hline System & $\boldsymbol{\Delta} \boldsymbol{G}_{\mathrm{vdw}}$ & $\boldsymbol{\Delta} \boldsymbol{E}_{\mathrm{MM}}$ & $\mathbf{- T \Delta} \boldsymbol{S}$ & $\boldsymbol{\Delta} \boldsymbol{G}_{\text {gas }}$ & $\boldsymbol{\Delta} \boldsymbol{G}_{\text {solv }}$ & $\boldsymbol{\Delta} \boldsymbol{G}_{\text {bind }}$ \\
\hline \hline Lact_BCDAmp & -134.8 & -273.3 & 74.1 & -199.2 & 56.6 & -142.6 \\
\hline Phen_BCDAmp & -113.3 & -225.4 & 69.6 & -155.8 & 60.3 & -95.5 \\
\hline Isob_BCDIbup & -96.3 & -168.3 & 61.2 & -107.2 & -7.9 & -115.1 \\
\hline Prop_BCDIbup & -93.0 & -208.9 & 65.6 & -143.3 & 45.5 & -97.8 \\
\hline
\end{tabular}
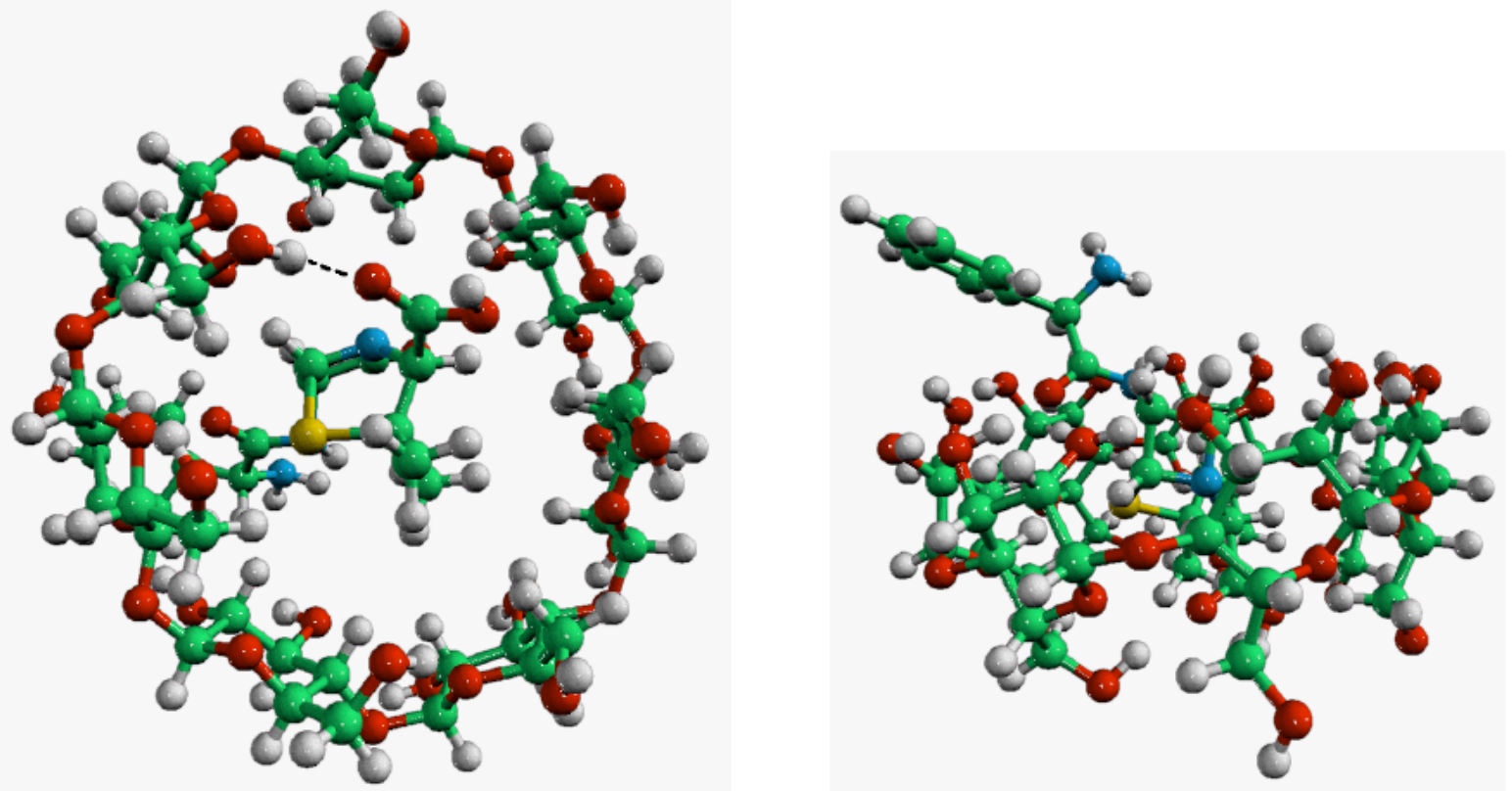

(a)
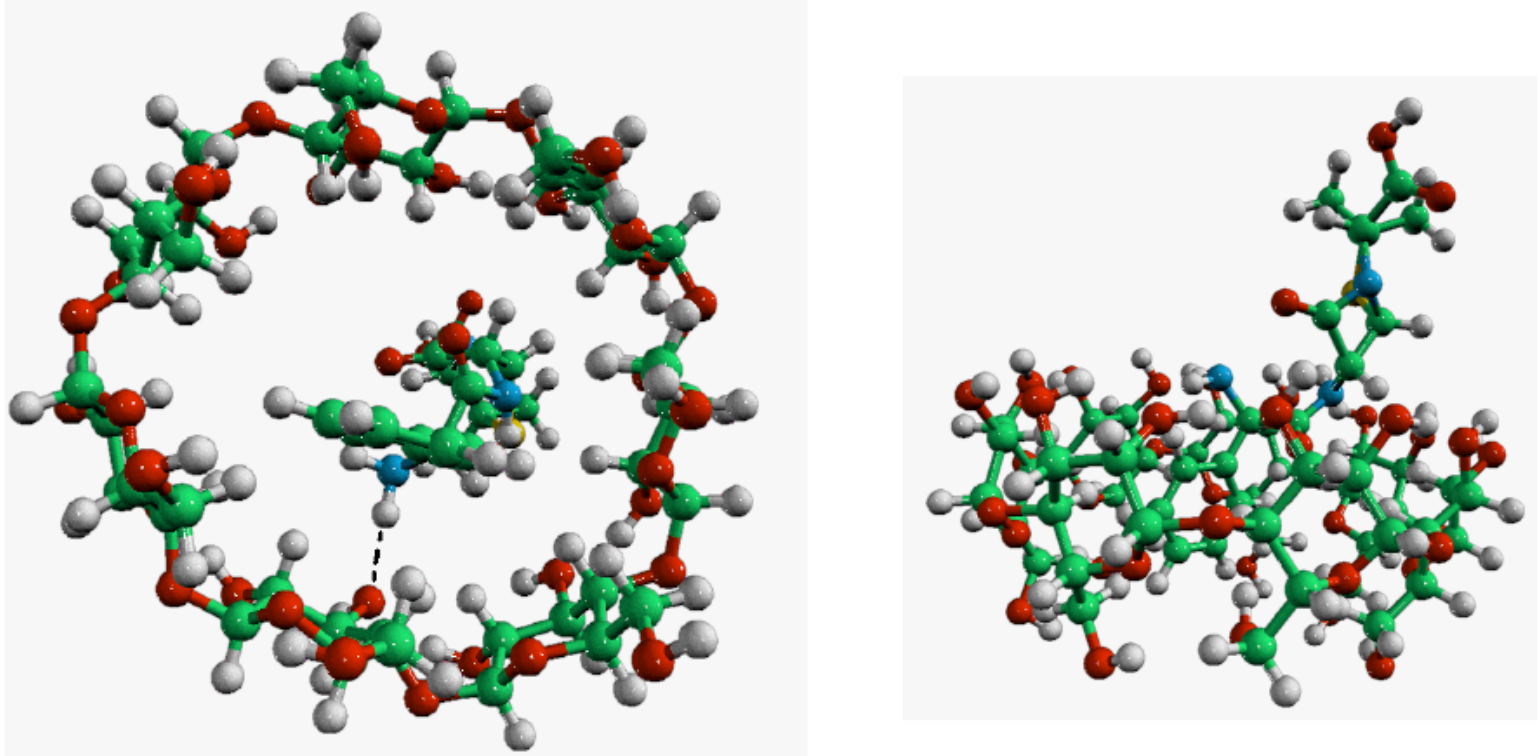

(b)

Figure 3: a. The average structures Lact_BCDAmp obtained using MM/PBSA analysis (without water molecules). Vertical view (left, dotted line represents the intermolecular hydrogen bond) and side view (right).

b. The average structures Phen_BCDAmp obtained using MM/PBSA analysis (without water molecules). Vertical view (left, dotted line represents the intermolecular hydrogen bond) and side view (right). 
shown in Table 2, the dispersion energy and interaction energy of Lact_BCDAmp are larger than those of Phen_BCDAmp; these results further clarify the large stabilization of Lact_BCDAmp.

\section{2. $\beta-C D+$ Ibuprofen}

Consideration of these inclusion phenomena and the MM/PBSA results shows that Isob_BCDIbup is more stabilized $\left(-17.3 \mathrm{~kJ} \mathrm{~mol}^{-1}\right)$ than Prop_BCDIbup and the van der Waals stabilization of Isob_BCDIbup is larger than that of Prop_BCDlbup. As shown in Figures 4a and 4b, ibuprofen in Isob_BCDIbup does not form intermolecular hydrogen bonds with the $\beta-C D$ cavity. Prop_BCDIbup appears to form an intermolecular hydrogen bond on the primary hydroxyl side of $\beta-C D$, with the $\mathrm{O}-\mathrm{H}$ distance of $0.09737 \mathrm{~nm}$, the $\mathrm{H} \cdots \mathrm{O}$ distance of $0.20505 \mathrm{~nm}$, and the $\mathrm{O}-\mathrm{H} \cdots \mathrm{O}$ angle of $168.651^{\circ}$. The interaction energy of Prop_BCDlbup is larger than that Isob_BCDlbup, as shown in Table 2. Conversely, the dispersion energy of Isob_BCDIbup is larger than that of Prop_BCDlbup. It seems that the affinity of the isobutyl group for the $\beta-C D$ cavity is


(a)
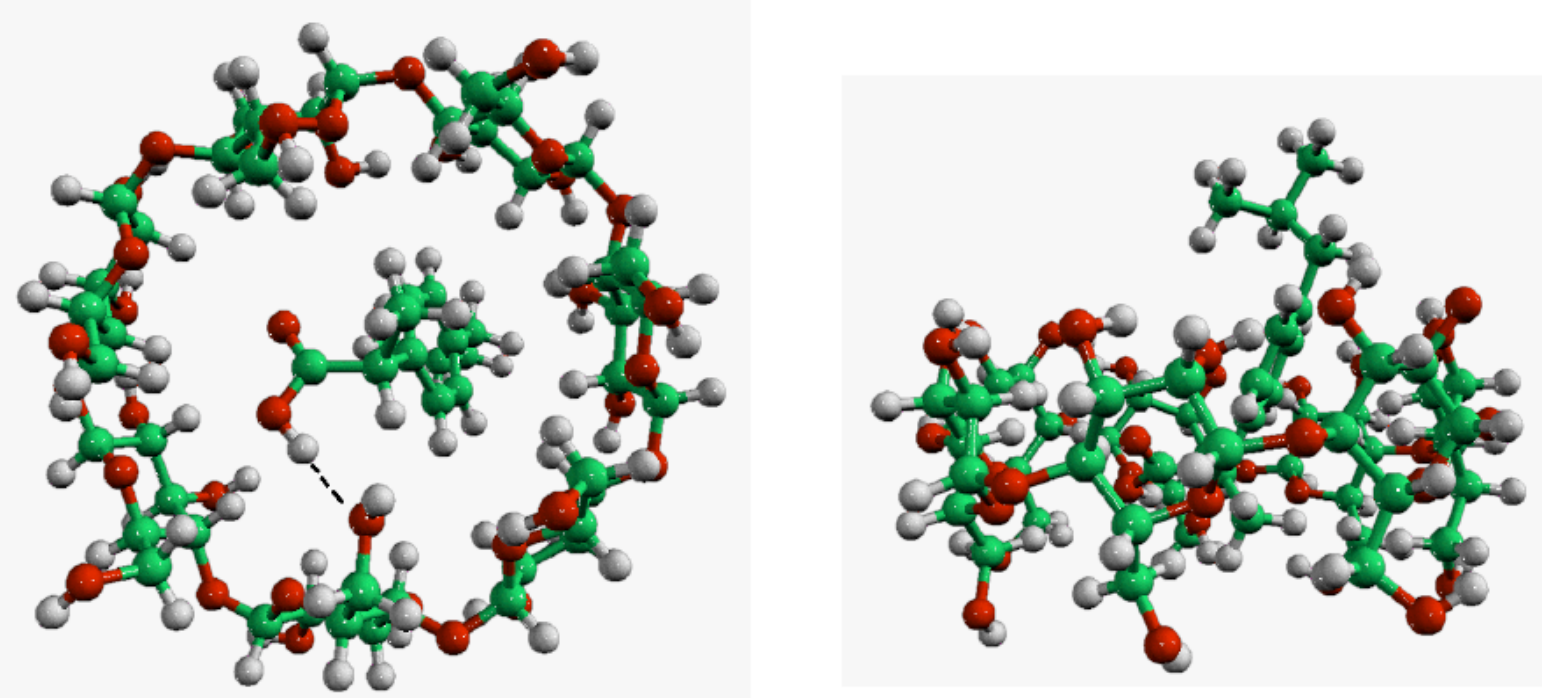

(b)

Figure 4: a. The average structures Isob_BCDlbup obtained using MM/PBSA analysis (without water molecules). Vertical view (left) and side view (right).

b. The average structures Prop_BCDlbup obtained using MM/PBSA analysis (without water molecules). Vertical view (left, dotted line represents the intermolecular hydrogen bond) and side view (right). 
Table 2: PIEDA Results for the Systems of $\beta-C D+$ Drug. All Data are Given in kJ mol ${ }^{-1}$

\begin{tabular}{|c|c|c|c|c|c|}
\hline System & $\boldsymbol{\Delta} \boldsymbol{G}_{\mathrm{vdw}}$ & $\boldsymbol{\Delta} \boldsymbol{E}_{\mathrm{MM}}$ & $\boldsymbol{- T \Delta} \mathbf{S}$ & $\Delta \boldsymbol{G}_{\text {gas }} \Delta \boldsymbol{G}_{\text {solv }}$ & $\boldsymbol{\Delta} \boldsymbol{G}_{\text {bind }}$ \\
\hline \hline Lact_BCDAmp & -237.3 & 430.6 & -93.7 & -206.3 & -106.7 \\
\hline Phen_BCDAmp & -109.4 & 290.0 & -92.7 & -156.3 & -137.4 \\
\hline Isob_BCDIbup & -42.0 & 61.8 & -33.9 & -132.7 & -151.5 \\
\hline Prop_BCDIbup & -97.2 & 67.7 & -43.6 & -205.8 \\
\hline
\end{tabular}

favorable since the inner walls of $\beta-C D$ are $\Delta E_{\text {internal }}$ considerably hydrophobic.

\section{CONCLUSION}

By considering these inclusion phenomena along with the MM/PBSA analysis results, we predict that a phenyl ring of ampicillin exists outside of the secondary hydroxyl side of $\beta-C D$ and that the lactam ring exists inside the $\beta-C D$ cavity. Further, we predict that the isobutyl group of ibuprofen exists inside the $\beta-C D$ cavity and that the propanoic acid exists outside of the secondary hydroxyl side of $\beta-C D$. It seems that the inclusion occurs to give the form in which the hydrophobic group of the drug is not exposed to water. These observations were borne out by the dispersion forces determined using PIEDA. Thus, the lactam ring of ampicillin was inside the $\beta-C D$ cavity in Lact_BCDAmp, which protected it from aggregation. This indicates that the inclusion complexes of ampicillin and $\beta-C D$ would be useful as drug delivery systems. The structures of the complexes could be predicted using two different methods that contain no empirical parameters. The experimental Gibbs energies of $\beta-C D$ + ampicillin[13] and $\beta-C D+$ ibuprofen[21] are $-17.3 \mathrm{~kJ}$ $\mathrm{mol}^{-1}$ and $-23.4 \mathrm{~kJ} \mathrm{~mol}^{-1}$, respectively. Thus, the theoretical values do not agree with experimental absolute values. In the future, we plan to perform thermodynamic integration calculations to quantitatively reveal the mechanism of interaction between $C D$ and drugs in aqueous solutions.

\section{APPENDIX}
$\Delta G_{\text {bind }}$
$=$ Binding free energy between $C D$ and a drug to form a complex
$\Delta E_{\mathrm{MM}} \quad=$ Change in the gas phase $\mathrm{MM}$ energy
$\Delta G_{\text {solv }} \quad=$ Solvation free energy
$-T \Delta S=$ Entropy term upon binding

$\Delta E_{\mathrm{els}}$

$\Delta E_{\mathrm{vdw}}$

$\Delta G_{\mathrm{PB}}$

$\Delta G_{S A}$

$\Delta G_{\text {gas }}$

$\Delta G_{\text {solv }}($ drug $)$

$\Delta G_{\text {solv }}(\beta-C D)$

$\Delta G_{\text {solv }}($ complex $)=$

$\Delta E_{\mathrm{ES}}$

$\Delta E_{\mathrm{EX}}$

$\Delta E_{\mathrm{CT}}$

$\Delta E_{\mathrm{DI}}$

\section{REFERENCES}

[1]

Takagi S, Kimura T, Maeda M. Some problems in solution microcalorimetry, experimental experiences by the authors, and the enthalpy-entropy compensation in cyclodextrin + alcohol inclusion-complex formation in aqueous solutions. Thermochim Acta 1985; 88: 247-54.

http://dx.doi.org/10.1016/0040-6031(85)85437-X

[2] Takagi S, Fujisawa M, Kimura T. Enthalpy and entropy changes on molecular inclusion of 1,3-butanediol into $\alpha$ and $\beta$-cyclodextrin cavities in aqueous solutions. Thermochim Acta 1991; 183: 289-97.

http://dx.doi.org/10.1016/0040-6031(91)80465-U

[3] Fujisawa M, Kimura T, Takagi S. The enthalpic stabilization on molecular inclusion of butanediol isomers into cyclodextrin cavities. Fluid Phase Equilib 1997; 136: 197-205. http://dx.doi.org/10.1016/S0378-3812(97)00120-9 
[4] Fujisawa M, Kimura T, Takagi S. Thermodynamic functions of molecular inclusion of some isomers of butanediol in gas phase into $\alpha$ - and $\beta$-cyclodextrin cavities in aqueous solutions at $298.15 \mathrm{~K}$. J Therm Anal Calorim 2001; 64: 14955. http://dx.doi.org/10.1023/A:1011584912731

[5] Fujisawa M, Kimura T. Enthalpy and entropy changes on molecular inclusion of 1-heptanol into $\alpha$ - and $\beta$-cyclodextrin cavities in aqueous solutions. Thermochim Acta 2004; 416: 51-4. http://dx.doi.org/10.1016/j.tca.2002.12.003

[6] Kimura T, Fujisawa M, Nakano $Y$, Kamiyama T, Otsu T, Maeda $\mathrm{M}$, et al. Calorimetric study on inclusion of some alcohols into $\alpha$-cyclodextrin cavities. Molecular mechanical calculation of hydration Gibbs energies. J Therm Anal Calorim 2007; 90: 581-5. http://dx.doi.org/10.1007/s10973-007-7914-1

[7] Kimura T, Fujie S, Yukiyama T, Fujisawa M, Kamiyama T, Aki $\mathrm{H}$. Enthalpy and entropy changes on molecular inclusion of pentane derivatives into $\alpha$-cyclodextrin cavities in aqueous solutions. J Inclusion Phenom Macrocyclic Chem 2011; 70: 269-78. http://dx.doi.org/10.1007/s10847-010-9877-2

[8] Kimura T, Yukiyama T, Fujisawa M. Thermodynamic properties of inclusion complexes of $\alpha$-cyclodextrin + aliphatic nitriles $\left(\mathrm{H}_{(}\left(\mathrm{CH}_{2}\right)_{n} \mathrm{CN}: \mathrm{n}=1-8\right)$ in aqueous solution. $\mathrm{J}$ Therm Anal Calorim 2012; 108: 695-704. http://dx.doi.org/10.1007/s10973-011-2144-y

[9] Rekharsky MV, Inoue Y. Complexation thermodynamics of cyclodextrins. Chem Rev 1998; 98: 1875-1918. http://dx.doi.org/10.1021/cr970015o

[10] Sun DZ, Wang SB, Wei XL, Yin BL. A microcalorimetric study of $\beta$-cyclodextrin with 3-alkoxyl-2-hydroxypropyl trimethyl ammonium bromides in aqueous solutions. J Chem Thermodyn 2005; 37: 431-6. http://dx.doi.org/10.1016/j.jct.2004.10.004

[11] Fujisawa M, Kimura T. Studies on molecular interactions of $\beta$-cyclodextrin and antiulcer agent. J Therm Anal Calorim 2006; 85: 589-91. http://dx.doi.org/10.1007/s10973-006-7656-5

[12] Aki H, Niiya $T$, Iwase $Y$, Kawasaki $Y$, Kumai K, Kimura T. Multimodal inclusion complexes of ampicillin with $\beta$ - cyclodextrins in aqueous solution. Thermochim Acta 2004; 416: 87-92.

http://dx.doi.org/10.1016/j.tca.2003.01.004

[13] Kollman PA, Massova I, Reyes C, Kuhn B, Huo S, Chong L, et al. Calculating structures and free energies of complex molecules: combining molecular mechanics and continuum models. Acc Chem Res 2000; 33: 889-97. http://dx.doi.org/10.1021/ar000033j

[14] Fedorov DG, Kitaura K. Pair interaction energy decomposition analysis. J Comput Chem 2007; 28: 222-37. http://dx.doi.org/10.1002/jcc.20496

[15] CONFLEX 6. CONFLEX Corporation 2006

[16] Frisch MJ, Trucks GW, Schlegel HB, Scuseria GE, Robb MA, Cheeseman JR, et al. Gaussian 09, Revision C.01. Gaussian, Inc., Wallingford CT 2009.

[17] Case DA, Darden TA, Cheatham III TE, Simmerling CL Wang J, Duke RE, et al. AMBER 12. University of California, San Francisco 2012.

[18] Ryckaert JP, Ciccotti G, Berendsen HJC. Numerical integration of the cartesian equations of motion of a system with constraints: molecular dynamics of n-alkanes. J Comput Phys 1977; 23: 327-41. http://dx.doi.org/10.1016/0021-9991(77)90098-5

[19] Wang J, Morin P, Wang W, Kollman PA. Use of MM-PBSA in reproducing the binding free energies to HIV-1 RT of TIBO derivatives and predicting the binding mode to HIV-1 RT of efavirenz by docking and MM-PBSA. J Am Chem Soc 2001; 123: $5221-30$ http://dx.doi.org/ 10.1021/ja003834q

[20] Schmidt MW, Baldridge KK, Boatz JA, Elbert ST, Gordon MS, Jensen $\mathrm{JH}$, et al. General atomic and molecular electronic structure system. J Comput Chem 1993; 14: 134763. http://dx.doi.org/10.1002/jcc.540141112

[21] Xing S, Zhang Q, Zhang C, Zhao Q, Ai H, Sun D. Isothermal titration calorimetry and theoretical studies on host-guest interaction of ibuprofen with $\alpha-, \beta$ - and $\gamma$-cyclodextrin. J Solution Chem 2009; 38: 531-43. http://dx.doi.org/10.1007/s10953-009-9394-3 